\title{
Dynamical Evolution of Trans-Neptunian Objects in High-Eccentricity Orbits
}

\author{
V.V. Emel'yanenko \\ South Ural University, Lenina 76, Chelyabinsk 454080, Russia
}

\begin{abstract}
The evolution of near-parabolic orbits with perihelia in the trans-neptunian region has been studied, considering the action of planetary, Galactic and stellar perturbations for the age of the Solar System. This investigation has led to the conclusion that the observed transneptunian objects in high-eccentricity orbits might originate from the inner core of the Oort cloud.
\end{abstract}

Recent discoveries of trans-neptunian objects in high-eccentricity orbits suggest that there exists a new class of small bodies in the outer Solar System. According to the information of the Minor Planet Center's WWW site (http://cfa-www.harvard.edu/iau/lists/Centaurs.html), now there are 6 objects with orbital eccentricities $e>0.5$ which have been observed at least in two oppositions: $1996 \mathrm{GQ}_{2} 1\left(q=38.0 \mathrm{AU}, e=0.59, i=13.4^{\circ}\right), 1996 \mathrm{TL}_{6} 6(q=35.0$ $\left.\mathrm{AU}, e=0.59, i=23.9^{\circ}\right), 1999 \mathrm{CY}_{1} 18\left(q=34.6 \mathrm{AU}, e=0.62, i=25.6^{\circ}\right), 1999 \mathrm{CZ}_{1} 18$ $\left(q=38.0 \mathrm{AU}, e=0.66, i=27.8^{\circ}\right), 1999 \mathrm{CF}_{1} 19\left(q=38.3 \mathrm{AU}, e=0.58, i=19.7^{\circ}\right), 1999$ $\mathrm{RZ}_{2} 15\left(q=31.0 \mathrm{AU}, e=0.70, i=25.5^{\circ}\right)$. Not only eccentricities are large, but the majority of these objects have perihelia located far from Neptune. Therefore it is difficult to explain their connection with bodies of the Edgeworth-Kuiper Belt. In this paper we investigate the possibility that these objects could come from the Oort cloud.

Comets injected by passing stars in the trans-neptunian region from the Oort cloud experience small planetary and Galactic perturbations. We follow thousands of such objects for up to $4 \mathrm{Gyr}$ as they evolve from near-parabolic orbits to short periods. Semi-major axes $a$ of initial orbits correspond to both inner and outer parts of the Oort cloud, and initial perihelion distances $q$ reach $10^{2} \mathrm{AU}$. We study the evolution of 1000 randomly oriented orbits for each value of $q$, their inclinations $i$ are distributed in the range $\left(0^{\circ}, 20^{\circ}\right)$.

We consider perturbations by the major planets Jupiter, Saturn, Uranus and Neptune on the basis of various methods. For near-parabolic orbits we use the analytical form of Keplerian mappings (Emel'yanenko 1992). The evolution of trans-neptunian orbits for which this approach is not valid is calculated by means of the Wisdom-Holman mapping method (Wisdom \& Holman 1991) improved to treat highly eccentric motion of small bodies. If both above-mentioned methods are not applicable (basically for orbits which are close to the planetary orbits), we start a numerical integration using Everhart's method (Everhart 1974).

We take into account perturbations from the core and the disc of the Galaxy. If $e$ is close to 1 , we apply the theory based on the analytical approximation for 
nearly rectilinear motion. Otherwise we use the first-order theory of perturbations which is similar to (Byl 1986).

We stop the calculations at one of the following conditions: (1) $a<0$; (2) $a<100 \mathrm{AU}$ and $q<31 \mathrm{AU}$; (3) the object has a close encounter with a star. The latter case is described as a random process with the probability $p_{s}=0.23 \times 10^{-15} a^{3.5}$ (see Hills 1981) of close encounters per revolution.

Table 1 presents results for the mean time $T$ and the frequency $s$ of comets in orbits with $a \sim 3000 \mathrm{AU}$ and different values of $q$ to evolve to orbits with $a<100 \mathrm{AU}$ and $q>31 \mathrm{AU}$.

Table 1. Frequencies and mean times of the evolution from orbits with $a \sim 3000 \mathrm{AU}$ to orbits with $a<100 \mathrm{AU}$ and $q>31 \mathrm{AU}$

\begin{tabular}{ccc}
$q(\mathrm{AU})$ & $s$ & $T(\mathrm{Myr})$ \\
\hline 30 & 0.003 & 516 \\
33 & 0.002 & 278 \\
36 & 0.003 & 852 \\
39 & 0.001 & 2753 \\
42 & 0.001 & 1521 \\
\hline \hline
\end{tabular}

There is a dependence of the frequency $s$ on initial semi-major axis: for instance, for orbits with $q=36 \mathrm{AU}$ our calculations give $s=0.012$ at $a=1000$ $\mathrm{AU}, s=0.002$ at $a=5000 \mathrm{AU}$ and $s=0$ at $a=10000 \mathrm{AU}$. Despite statistical errors these results show that the probability of the transfer of trans-neptunian comets from orbits with semi-major axes which are typical for the inner core of the Oort cloud to orbits with $a<100 \mathrm{AU}$ is quite large, and the observed transneptunian objects in high-eccentricity orbits might originate from the inner core of the Oort cloud.

Acknowledgments. This work was supported by RFBR Grant 98-02-17918. The author acknowledges support from an IAU grant to attend the 24th General Assembly.

\section{References}

Byl, J. 1986, Earth, Moon, Planets, 36, 263

Emel'yanenko, V.V. 1992, Celest. Mech. Dynam. Astron., 54, 91

Everhart, E. 1974, Celest. Mech., 10, 35

Hills, J.G. 1981, AJ, 86, 1730

Wisdom, J., \& Holman, M. 1991, AJ, 102, 1528 